Polymer Journal, Vol. 38, No. 11, pp. 1152-1159 (2006)

(C) 2006 The Society of Polymer Science, Japan

\title{
Conformational Analysis of Poly(propionaldehyde azine) in Dilute Solution
}

\author{
Akihito HASHIDZUME, Yusuke UENO, and Takahiro SATO ${ }^{\dagger}$ \\ Department of Macromolecular Science, Graduate School of Science, Osaka University, \\ 1-1 Machikaneyama-cho, Toyonaka 560-0043, Japan
}

(Received June 30, 2006; Accepted August 22, 2006; Published October 4, 2006)

\begin{abstract}
The conformation of a photoresponsive polymer bearing azo units in the main chain, poly(propionaldehyde azine) (PrAz), in tetrahydrofuran was investigated by viscosity measurements before and after trans-to-cis photoisomerization and also by the rotational isomeric state (RIS) model. Before photoisomerization (i.e., in the case of trans-pPrAz), viscosity data indicated that pPrAz took a more extended conformation than did a diene polymer ana$\log$ poly(butadiene). The RIS model analysis revealed that the extended conformation of pPrAz arose mainly from the difficulty of gauche conformation around the carbon-carbon bond in the main chain. After trans-to-cis photoisomerization (i.e., in the case of pPrAz consisting of trans and cis units), viscosity data indicated that the photoisomerization led to a slight extension of the pPrAz chain. The RIS model analysis explained that the photoisomerization formed preferentially the anti-cis isomer, resulting in a larger characteristic ratio to avoid severe steric hindrance between the ethyl groups on neighboring main-chain carbon atoms. [doi:10.1295/polymj.PJ2006066]

KEY WORDS Conformational Analysis / Characteristic Ratio / Viscometry / Sedimentation Equilibrium / Poly(propionaldehyde azine) / Photoisomerization /
\end{abstract}

Materials responsive to external stimuli may be useful for molecular machines and motors and thus they have been attracting increasing interest of researchers from the viewpoint of nanotechnological applications. ${ }^{1}$ Among stimuli responsive materials, photoresponsive ones may be most promising because of their fast response. ${ }^{2}$ Pioneering works by Irie et $a l^{3,4}$ have demonstrated that polymers bearing azobenzene moieties in their main chain undergo reversible trans-to-cis and cis-to-trans isomerization under photoirradiation to cause repetitive changes in their solution viscosity: trans-to-cis photoisomerization leads a decrease in solution viscosity whereas cis-to-trans photoisomerization returns the solution viscosity.

Kamachi et $a l .{ }^{5-7}$ have reported that azine compounds, diene analogs bearing $\mathrm{C}=\mathrm{N}$ bond, are polymerized with Grignard reagents to form polymers consisting of 1,4-trans units. Their preliminary results have also indicated that these polymers of azine compounds undergo photoisomerization under UV irradiation, leading to changes in the polymer conformation in solution. ${ }^{8}$

To our best knowledge, however, there have been no detail studies on conformational change of photoresponsive polymers upon photoisomerization although it may be important for molecular design of photoresponsive polymers with high performance. Thus, we studied the conformation of poly(propionaldehyde azine) (pPrAz) in solution before and after<smiles>CC(C)N=NC1(C)CCCCC1(C)C</smiles>

Scheme 1. Chemical structure of pPrAz.

photoisomerization by viscometry and also by the rotational isomeric state model. Moreover, since azine polymers are diene polymer analogs, we compared the conformation of pPrAz with those of poly(butadiene) and poly(isoprene) with the trans and cis configurations to argue the effect of the replacement of the carbon double bond by the nitrogen one on the chain conformation.

\section{EXPERIMENTAL}

\section{Materials}

A commercially available solution of methylmagnesium iodide $\left(\mathrm{CH}_{3} \mathrm{MgI}\right)$ ( $\mathrm{ca} .2 \mathrm{M}$ solution in diethyl ether, TCI) was put into an ampule with a syringe under an argon atmosphere. Tetrahydrofuran (THF) used for measurements was purified by atmospheric distillation. Other reagents were used without further purification.

Propionaldehyde azine (PrAz) was prepared from propionaldehyde and hydrazine monohydrate according to the procedure of Curtius and Zinkeisen. ${ }^{9} \mathrm{PrAz}$

${ }^{\dagger}$ To whom correspondence should be addressed (Tel \& Fax: +81-6-6850-5461, E-mail: tsato@chem.sci.osaka-u.ac.jp). 
was purified by successive distillations over calcium hydride under an argon atmosphere and stored under an argon atmosphere.

Polymer samples of $\operatorname{PrAz}(\mathrm{pPrAz})$ were prepared by anionic bulk polymerization initiated by $\mathrm{CH}_{3} \mathrm{MgI}$ under an argon atmosphere according to the procedure of Kamachi et al. ${ }^{5-7}$ The polymers obtained were purified by reprecipitation three times from THF solution to excess methanol. Polymers were recovered by freeze-drying from benzene solution.

\section{Photoisomerization of $\mathrm{pPrAz}$}

Solutions of pPrAz in THF were irradiated with an Iuchi handy UV lamp SLUV-6 $(365 \mathrm{~nm})$. The distance between the sample cell and the lamp was fixed at $c a$. $11 \mathrm{~cm}$. The polymers obtained were purified by reprecipitation three times from THF solution to excess methanol. Polymers were recovered by freeze-drying from benzene solution.

\section{Measurements}

Sedimentation equilibrium measurements were performed for solutions of pPrAz samples in THF at $25^{\circ} \mathrm{C}$ using a Beckman-Coulter Optima XL-I ultracentrifuge equipped with a Rayleigh interferometer with a $675 \mathrm{~nm}$ light emitting from a diode laser. Aluminum $12 \mathrm{~mm}$ double-sector cells were used, and the height of the solution column was adjusted to $c a$. $2.5 \mathrm{~mm}$. The rotor speed was fixed at 35,000 rpm.

The apparent molecular weight $M_{\text {app }}$ was calculated by ${ }^{10,11}$

$$
M_{\mathrm{app}}=\frac{2 R T\left(c_{\mathrm{b}}-c_{\mathrm{a}}\right)}{\omega^{2}\left(\boldsymbol{r}_{\mathrm{b}}^{2}-\boldsymbol{r}_{\mathrm{a}}^{2}\right) c_{0}\left(1-\bar{v} \rho_{0}\right)}
$$

where $\boldsymbol{r}_{\mathrm{a}}$ and $\boldsymbol{r}_{\mathrm{b}}$ are the distances from the center of revolution to the meniscus and cell bottom, respectively, and $c_{\mathrm{a}}$ and $c_{\mathrm{b}}$ are polymer mass concentrations at $\boldsymbol{r}_{\mathrm{a}}$ and $\boldsymbol{r}_{\mathrm{b}}$ respectively, under the centrifugal field; $\omega$ is the angular velocity, $c_{0}$ is the mass concentration of the solution at $\omega=0, \bar{v}$ is the partial specific volume, $\rho_{0}$ is the solvent density, and $R T$ is the gas constant multiplied by the absolute temperature.

The weight-average molecular weight $M_{\mathrm{w}}$ and the second virial coefficient $A_{2}$ were determined from the plot of $M_{\text {app }}{ }^{-1}$ versus the average concentration $\bar{c} \equiv$ $\left(c_{\mathrm{a}}+c_{\mathrm{b}}\right) / 2$ in a low concentration region using ${ }^{10,11}$

$$
\frac{1}{M_{\mathrm{app}}}=\frac{1}{M_{\mathrm{w}}}+2 A_{2} \bar{c}+\cdots
$$

Densities $\rho$ of pure THF and THF solutions of pPrAz with different concentrations were measured at $25^{\circ} \mathrm{C}$ using an Anton-Paar DMA 5000 densitometer to determine $\bar{v}$ and $\rho_{0}$. The concentrations $c_{\mathrm{a}}$ and $c_{\mathrm{b}}$ under the centrifugal field were determined by interferometry along with the specific refractive index in- crement $\partial n / \partial c$. Values of $\partial n / \partial c$ at 436 and $546 \mathrm{~nm}$ were measured at $25^{\circ} \mathrm{C}$ using a modified SchultzCantow type differential refractometer, and extrapolated to obtain the $\partial n / \partial c$ value at $675 \mathrm{~nm}$.

Viscosities of THF solutions of pPrAz samples were measured at $25^{\circ} \mathrm{C}$ using a conventional capillary viscometer of the Ubbelohde type. The intrinsic viscosity $[\eta]$ and the Huggins coefficient $k^{\prime}$ were determined using the Huggins and Mead-Fuoss plots.

${ }^{1} \mathrm{H}$ NMR spectra were obtained on a JEOL JNM EX270 spectrometer in $\mathrm{CDCl}_{3}$ at $30^{\circ} \mathrm{C}$. UV-vis absorption spectra were recorded on a JASCO UV-520 spectrophotometer in THF.

\section{RESULTS AND DISCUSSION}

\section{Basic Characteristics of pPrAz Samples Used in This Study}

The pPrAz samples employed in this study were prepared by anionic bulk polymerization initiated by $\mathrm{CH}_{3} \mathrm{MgI}$ according to the procedure of Kamachi et al. ${ }^{5-7}$ Kamachi et al. $^{5-7}$ have reported that polymers of alkyl aldehyde azines obtained using Grignard reagents are composed of 1,4-trans units, and also suggested that the azine polymers contain both erythro and threo units (Figure 1). Fractions of 1,4-trans, erythro, and threo units in the pPrAz samples used in this study were estimated by ${ }^{1} \mathrm{H}$ NMR spectroscopy (Figure 2a). While the trans fraction was almost unity, the fractions of erythro and threo units were $c a$. 0.65 and 0.35 , respectively, for all the samples. ${ }^{12}$

Weight-average molecular weights $M_{\mathrm{w}}$ and second virial coefficients $A_{2}$ for the pPrAz samples were determined by sedimentation equilibrium. Figure $3 \mathrm{a}$ demonstrates $M_{\text {app }}{ }^{-1}$ as a function of $\bar{c}$. All the series of plots exhibit good linear relationships. From intercepts and slopes of the straight lines, $M_{\mathrm{w}}$ and $A_{2}$ values were determined as listed in Table I. All pPrAz

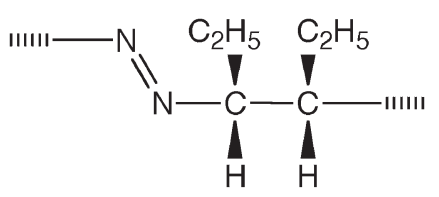

erythro

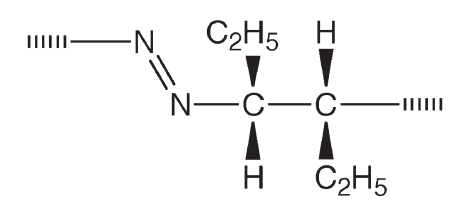

threo

Figure 1. Molecular structures of erythro- and threo-transpPrAz. 
${ }^{2} \mathrm{~V}$ บ! วถินеบว

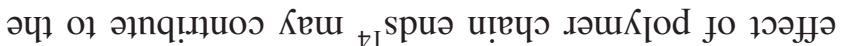

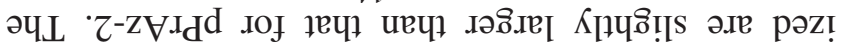
-ләшо

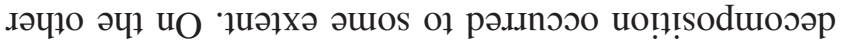

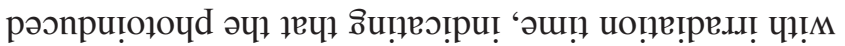

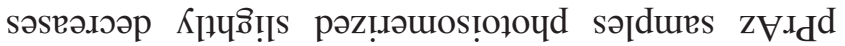

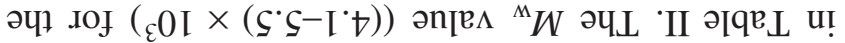

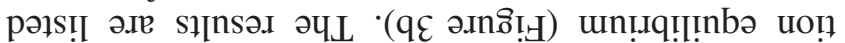

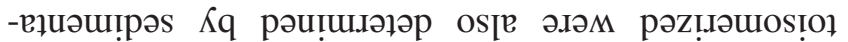

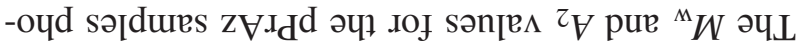

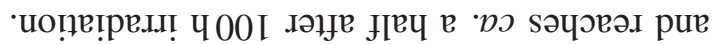

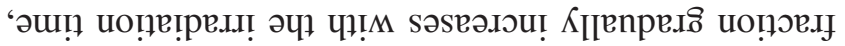

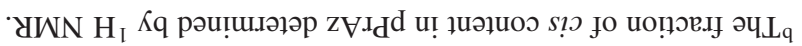

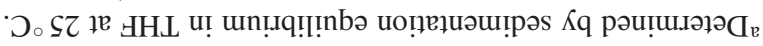

\begin{tabular}{|c|c|c|c|c|}
\hline $0^{\circ} \mathcal{\varepsilon}$ & $L^{\prime} t$ & $S^{\circ}{ }^{\circ} 0$ & SOI & t.uIZZ-ZVIId \\
\hline $8^{\circ} t$ & $I^{\prime} t$ & $8 \varepsilon^{\circ} 0$ & 09 & $\mathcal{E} \cdot \mathrm{uI}_{\tau-Z}-\mathrm{Z} \cdot \mathrm{I}_{\mathbf{d}} \mathrm{d}$ \\
\hline $8 \cdot z$ & $t^{\circ} \varsigma$ & $6 Z^{\circ} 0$ & $8 t$ & Z.u!z-ZV.Idd \\
\hline$I^{\circ} \mathcal{E}$ & $\varsigma \varsigma$ & $\varepsilon \varepsilon^{\circ} 0$ & $\nabla \tau$ & {$\left[\cdot . u ! z-Z \forall \cdot I_{d} d\right.$} \\
\hline 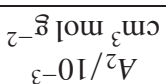 & ${ }_{\varepsilon} 0 \mathrm{I} /{ }^{M} W$ & $q^{s ! \rho} f$ & 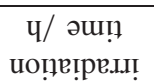 & әроэ ләшК Коd \\
\hline
\end{tabular}

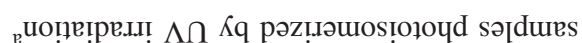
$\mathrm{Z}_{\mathrm{V}} \mathrm{Id}_{\mathrm{d}}^{\mathrm{d}}$ әчl .

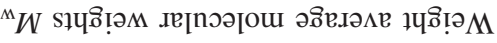

'II əIq'e $\mathbf{L}$

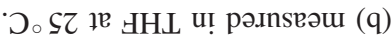

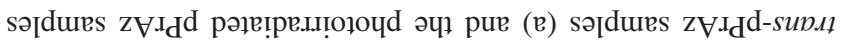

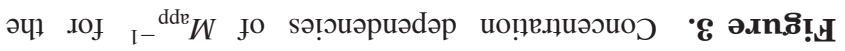

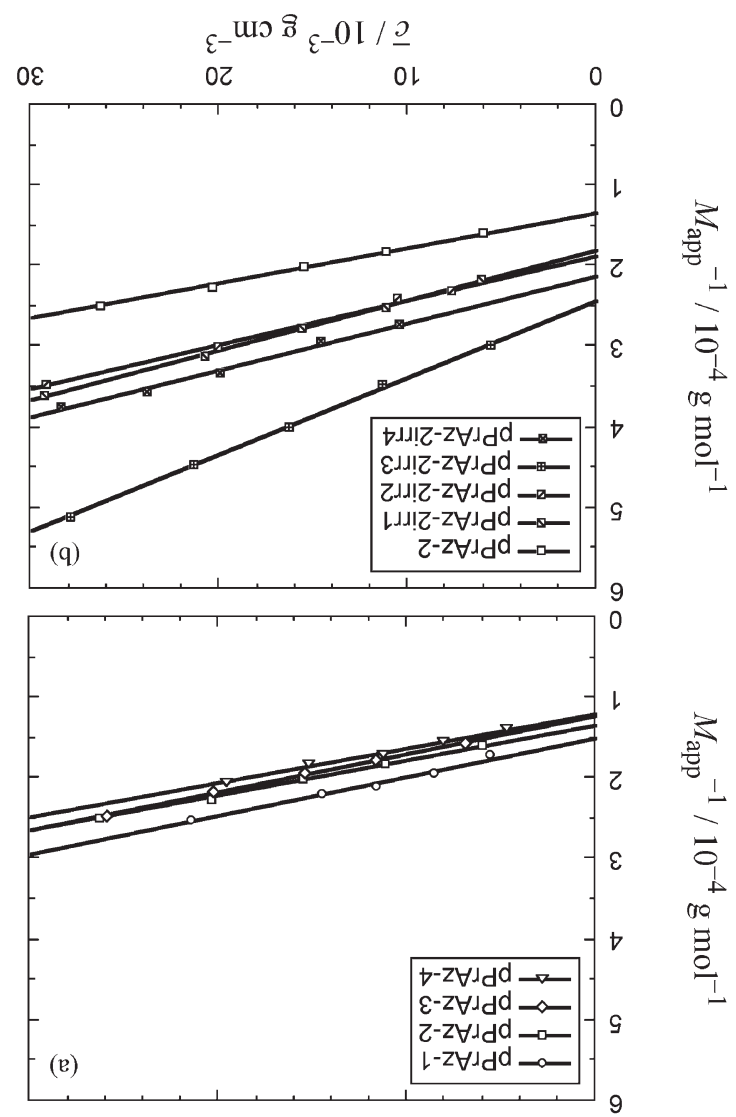

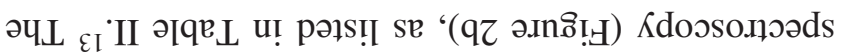

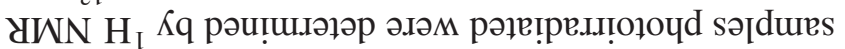

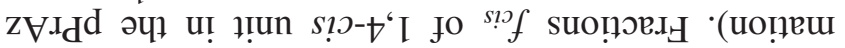

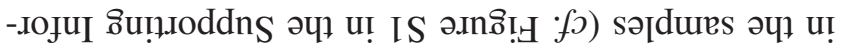

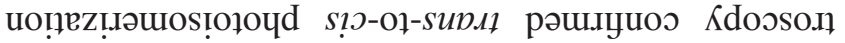

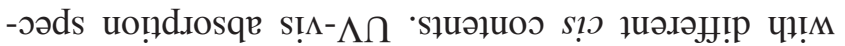

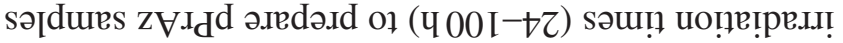

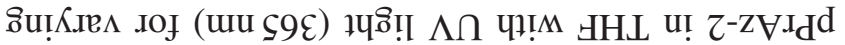

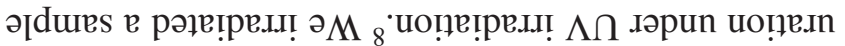

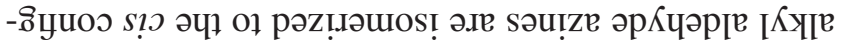

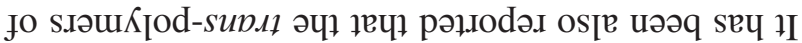

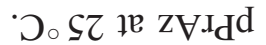

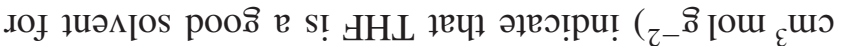

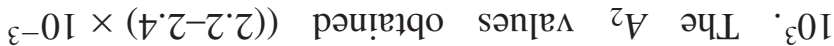

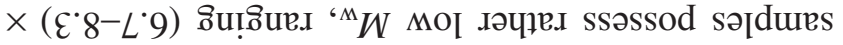

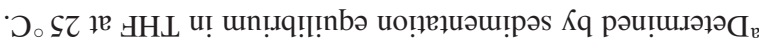

\begin{tabular}{|c|c|c|}
\hline$\tau \cdot \tau$ & $\varepsilon 8$ & $t^{-Z} \forall \cdot I_{d}^{d}$ \\
\hline$t \cdot \tau$ & I'8 & $\mathcal{E}-Z_{\forall} \cdot I_{d} d$ \\
\hline$\tau \cdot \tau$ & $\varsigma L$ & $z-Z \forall \cdot I_{d} d$ \\
\hline$t r$ & $L \cdot 9$ & $I^{-Z} Z \cdot{ }^{d} d$ \\
\hline 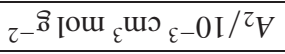 & ${ }_{\varepsilon} 0 \mathrm{I} /{ }^{M} W$ & әроэ ләшК Цоd \\
\hline
\end{tabular}

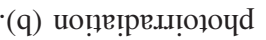

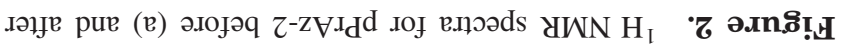

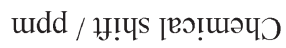

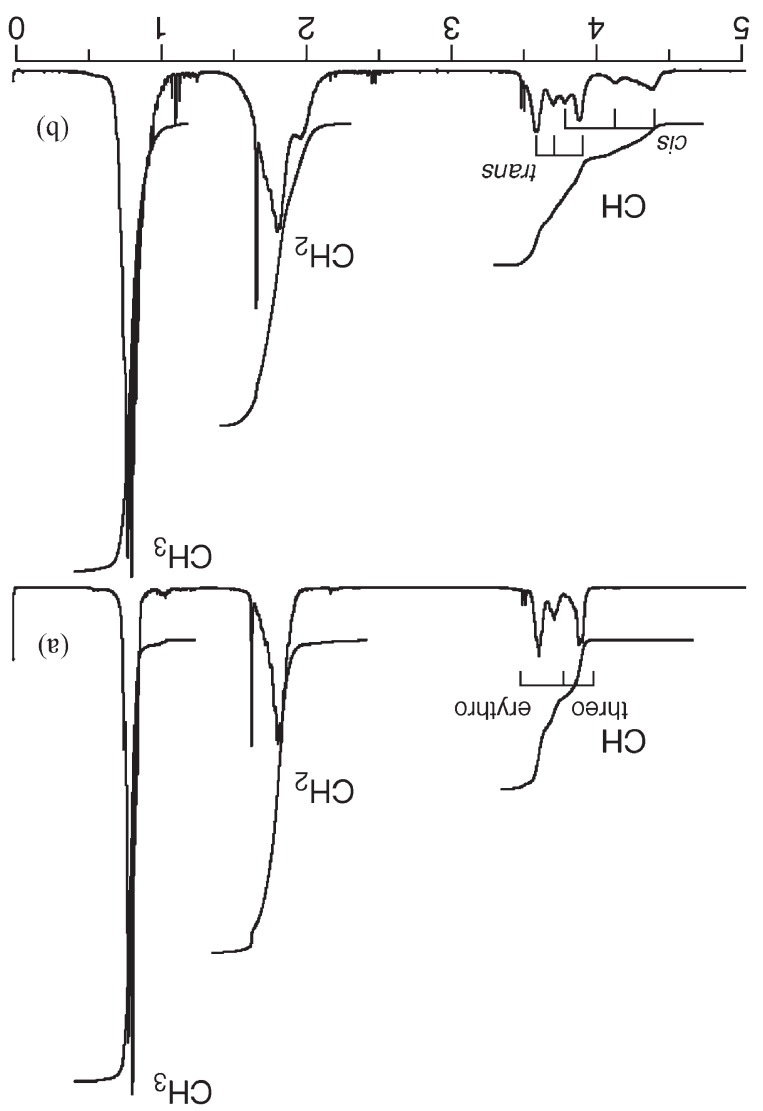




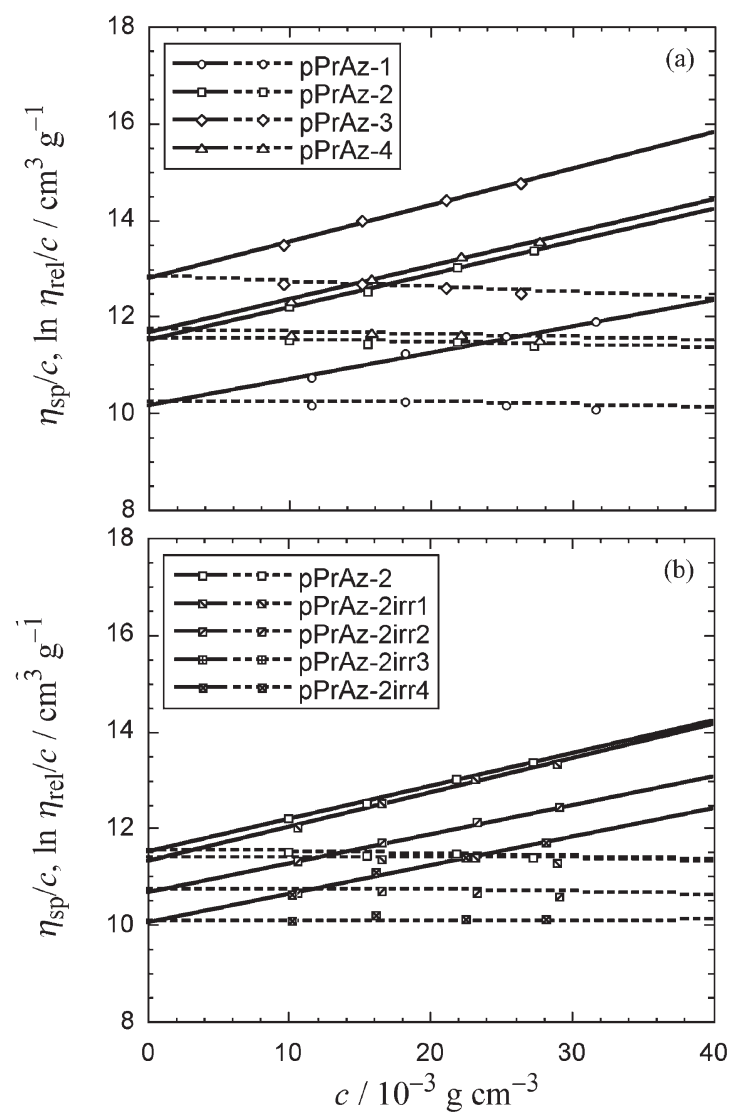

Figure 4. Huggins and Mead-Fuoss plots for (a) trans-pPrAz samples and (b) pPrAz sample photoirradiated in THF. Here, $\eta_{\mathrm{sp}}$ and $\eta_{\text {rel }}$ are the specific and relative viscosities of the solution.

Table III. Intrinsic viscosities [ $\eta]$, Huggins constants $k^{\prime}$, and charateric ratios $C_{N}$ for the pPrAz samples

\begin{tabular}{lccc}
\hline polymer code & {$[\eta] / \mathrm{cm}^{3} \mathrm{~g}^{-1}$} & $k^{\prime}$ & $C_{N}$ \\
\hline pPrAz-1 & 10.2 & 0.49 & 8.42 \\
pPrAz-2 & 11.5 & 0.48 & 8.78 \\
pPrAz-3 & 12.8 & 0.44 & 9.20 \\
pPrAz-4 & 11.7 & 0.48 & 8.60 \\
pPrAz-2irr1 & 11.4 & 0.51 & 9.69 \\
pPrAz-2irr2 & 10.7 & 0.49 & 9.34 \\
pPrAz-2irr3 & 11.2 & 0.52 & 10.56 \\
pPrAz-2irr4 & 10.1 & 0.54 & 9.40 \\
\hline
\end{tabular}

\section{Viscosity Measurements}

Figure 4 demonstrates Huggins and Mead-Fuoss plots for the pPrAz samples in $\mathrm{THF}$ at $25^{\circ} \mathrm{C}$. For all the pPrAz samples, plots exhibit good linear relationships. From intercepts and slopes of the straight lines, $[\eta]$ and $k^{\prime}$ values were determined as listed in Table III. Values of $k^{\prime}$ for all the pPrAz samples are within a normal range.

Figure 5 plots those $[\eta]$ results against the degree of polymerization $N_{\mathrm{w}}$ calculated by $M_{\mathrm{w}} / M_{0}$ with the molar mass $M_{0}$ of the monomer unit. Although $[\eta]$ values are not so different before and after photoisomerization, the data points (filled circles) after photo-

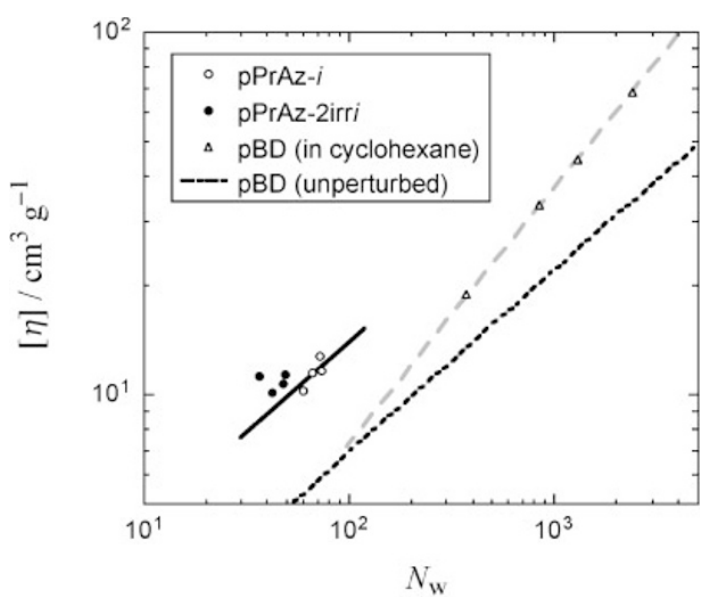

Figure 5. Comparison of $[\eta]$ for pPrAz before and after photoisomerization in THF as well as for $\mathrm{pBD}$ in the perturbed and unperturbed states. Triangles and the dotted line represent Colby et al.'s data ${ }^{15}$ in cyclohexane and calculated values using Mark's characteristic ratio, ${ }^{17}$ respectively.

isomerization locate appreciably above the line passing through the data points (unfilled circles) for trans-pPrAz with a slope of 0.5 . This indicates that the trans-to-cis photoisomerization extends the pPrAz chain. (If the chain dimension of pPrAz might be affected by the excluded volume effect, the filled circles should obey a steeper line, which deviates more from the triangles.)

The change in the chain dimension upon photoirradiation for this azine polymer is much less remarkable than that for polymers bearing azobenzene moieties in their main chain. ${ }^{3,4}$ Moreover, it is amazing that the trans-to-cis photoisomerization changes the chain dimension oppositely between the azine and azobenzene polymers.

In Figure 5, triangles represent $[\eta]$ data for poly(butadiene) (pBD) with a cis content of $c a .0 .4$ in cyclohexane (a good solvent) reported by Colby et al. ${ }^{15}$ To compare the chain dimension for the two different polymers, Colby et al.'s [ $\eta$ ] were multiplied by the ratio of $M_{0}$ of $\mathrm{pBD}$ to that of pPrAz in the plot. Though $N_{\mathrm{w}}$ ranges investigated for the two polymers do not overlap, the extrapolation of Colby et al.'s data definitely indicates that the chain dimension of $\mathrm{pPrAz}$ is larger than that of pBD at the same $N_{\mathrm{w}}$.

The dotted line in the same figure indicates $[\eta]$ (multiplied by the $M_{0}$ ratio) for $\mathrm{pBD}$ with $f_{\text {cis }}=0.4$ in the unperturbed state, calculated by the equation

$$
[\eta]=\Phi\left(C_{N} \cdot 4 N \overline{b^{2}}\right)^{3 / 2} / M
$$

where $\Phi$ is the Flory viscosity constant, $C_{N}$ is the characteristic ratio, $\overline{b^{2}}$ is the square average bond length of the polymer main chain, and $M$ and $N$ are the polymer molecular weight and degree of polymerization, respectively; for $\mathrm{pBD} \overline{b^{2}}=0.0221 \mathrm{~nm}^{2}$. The characteris- 
tic ratio $C_{N}$ for pBD with $f_{\text {cis }}=0.4$ was calculated to be 5.2 at infinite $N$, using the rotational isomeric state (RIS) model with parameters used by Mark ${ }^{16,17}$ (see below), and $\Phi$ was chosen to be $2.5 \times 10^{23} \mathrm{~mol}^{-1}{ }^{18}$ Using this theoretical line, the viscosity expansion factor $\alpha_{\eta}{ }^{3}$ for Colby et al.'s lowest molecular weight pBD sample $\left(N_{\mathrm{w}}=370\right)$ in cyclohexane was estimated to be as small as 1.4, and the extrapolation of Colby et al.'s $[\eta]$ data implies that the excluded volume effect vanishes around $N_{\mathrm{w}}=100$.

Since we can expect that the strength of the excluded volume for $\mathrm{pPrAz}$ in THF is similar to that of $\mathrm{pBD}$ in cyclohexane from $A_{2}$ data, and moreover that the more extended conformation of pPrAz weakens the excluded volume effect, our pPrAz samples with $N_{\text {w }}<100$ used in this study may be free from the excluded volume effect. Thus, eq 3 for the unperturbed chain can be applied for $[\eta]$ data of our pPrAz samples. For pPrAz, $\overline{b^{2}}$ is $0.0210 \mathrm{~nm}^{2}$ (see below), and we can estimate $C_{N}$ from eq 3 and experimental $[\eta]$ for trans-pPrAz and photoisomerized one. The results, listed in Table III, are larger than $C_{\infty}\left(C_{N}\right.$ at infinite $N)$ not only for trans-pBD $(=5.8)$ but also for transpoly(isoprene) (pIP) (=7.35).

\section{Analysis Using the Rotational Isomeric State (RIS) Model}

Mark ${ }^{16,17}$ calculated characteristic ratios for diene polymers, pBD and pIP, on the basis of the RIS model, and compared these calculated values with experimental results. We here calculated $C_{N}$ for pPrAz, referring to the procedure of Mark. Let us first consider trans-pPrAz. Figure 6 shows the chemical structure of the repeat unit of erythro-trans-pPrAz, where the skeletal atoms $\mathrm{N}, \mathrm{N}, \mathrm{C}, \mathrm{C}$, and $\mathrm{N}$ are defined as atoms $0,1,2,3$, and 4 , respectively, the bond length between atoms $i-1$ and $i$ are denoted as $b_{i}$, and an angle formed by bonds $i$ and $i+1$ as $\theta_{i}$. This numbering is the same as Mark's for the diene analogues. Bond lengths and angles in Figure 6 were estimated using a CambridgeSoft Chem3D Ultra software (version 10.0) as follows: $b_{1}=0.125 \mathrm{~nm} ; b_{2}=b_{4}=0.149 \mathrm{~nm} ; b_{3}=$ $0.155 \mathrm{~nm} ; \theta_{1}=\theta_{4}=113.4^{\circ} ; \theta_{2}=\theta_{3}=107.3^{\circ}$. These parameters are slightly different from the correspond-

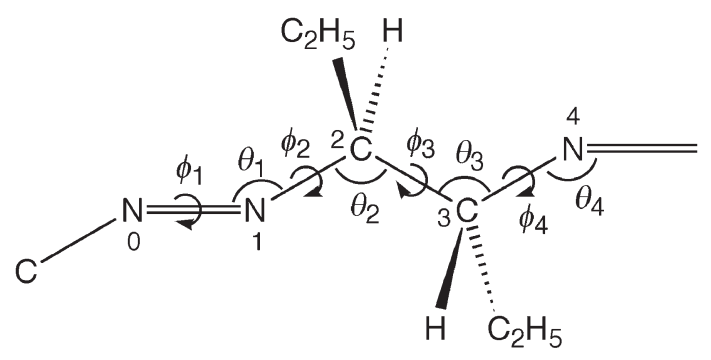

Figure 6. Molecular structure of erythro-trans-pPrAz. ing parameters for the diene polymer analogues.

In the RIS approximation, each skeletal bond adopts one of a small number of discrete rotational states, where the rotational angle about bond $i$ is defined as $\phi_{i}$ measured from the trans state of $0^{\circ}$. Here, we consider the same rotational isomers as those Mark considered for the diene polymer analogues, explained as follows. Since bond 1 is double bond, $\phi_{1}$ is fixed at $0^{\circ}$. Bonds 2 and 4 are single bonds lying between double and single bonds, and thus $\phi_{2}$ and $\phi_{4}$ can be assumed to be $\pm 60^{\circ}$ and $180^{\circ}$ by its chemical bond nature. ${ }^{19-21}$ The single bond 3 between two single bonds may adopt trans and gauche states (i.e., $\phi_{3}=0^{\circ}$, $\left.\pm 120^{\circ}\right)$ to avoid the steric repulsion between atoms attached to $\mathrm{C}^{2}$ and $\mathrm{C}^{3}$. Thus there are $27\left(=3^{3}\right)$ rotational isomers per monomer unit in the RIS model for trans-pPrAz.

For the rotational states for bond 2, statistical weights of $\phi_{2}= \pm 60^{\circ}$ and $180^{\circ}$ are defined as 1 and $\alpha$, respectively. Mark ${ }^{17}$ introduced the following two statistical weight matrices with respect to rotations around bonds 3 and 4 of trans-diene polymers,

\begin{tabular}{crrcc}
\hline & & \multicolumn{3}{c}{$\phi_{3}$} \\
\cline { 3 - 5 } & & $0^{\circ}$ & $120^{\circ}$ & $-120^{\circ}$ \\
\hline$\phi_{2}$ & $60^{\circ}$ & 1 & $\sigma$ & $\sigma$ \\
& $180^{\circ}$ & 1 & $\sigma \beta$ & $\sigma \beta$ \\
& $-60^{\circ}$ & 1 & $\sigma$ & $\sigma$ \\
\hline & & \multicolumn{3}{c}{$\phi_{4}$} \\
\cline { 3 - 5 } & & $60^{\circ}$ & $180^{\circ}$ & $-60^{\circ}$ \\
\hline$\phi_{3}$ & $0^{\circ}$ & 1 & $\alpha$ & 1 \\
& $120^{\circ}$ & 1 & $\alpha \beta$ & 1 \\
& $-120^{\circ}$ & 1 & $\alpha \beta$ & 1 \\
\hline
\end{tabular}

where statistical weights of $\phi_{3}=0^{\circ}$ and $120^{\circ}$ are defined as 1 and $\sigma$, respectively. When bond 2 or 4 is in the cis state $\left(\phi_{i}=180^{\circ}, i=2\right.$ or 4$)$, bond 3 may take less favorably the gauche states, which is taken into account by a factor $\beta(<1)$ in the above matrices.

On the other hand, the monomer unit of trans-pPrAz has two ethyl groups on the neighboring $\mathrm{C}^{2}$ and $\mathrm{C}^{3}$ atoms which affect the rotation around bond 3 . As shown in Figure 7, the two ethyl groups come closer at $\phi_{3}= \pm 120^{\circ}$ for the erythro unit and at $\phi_{3}=0^{\circ}$ and $120^{\circ}$ for the threo unit (the R,R-form), so that statistical weights for such rotational states should be multiplied by a factor $\gamma(<1)$, which was not necessary for diene polymers. As a result, the above upper matrix for trans-diene polymers should be replaced by

\begin{tabular}{ccccc}
\hline \multirow{2}{*}{ erythro } & & \multicolumn{3}{c}{$\phi_{3}$} \\
\cline { 3 - 5 } & & $0^{\circ}$ & $120^{\circ}$ & $-120^{\circ}$ \\
\hline$\phi_{2}$ & $60^{\circ}$ & 1 & $\sigma \gamma$ & $\sigma \gamma$ \\
& $180^{\circ}$ & 1 & $\sigma \beta \gamma$ & $\sigma \beta \gamma$ \\
& $-60^{\circ}$ & 1 & $\sigma \gamma$ & $\sigma \gamma$ \\
\hline
\end{tabular}


(a)

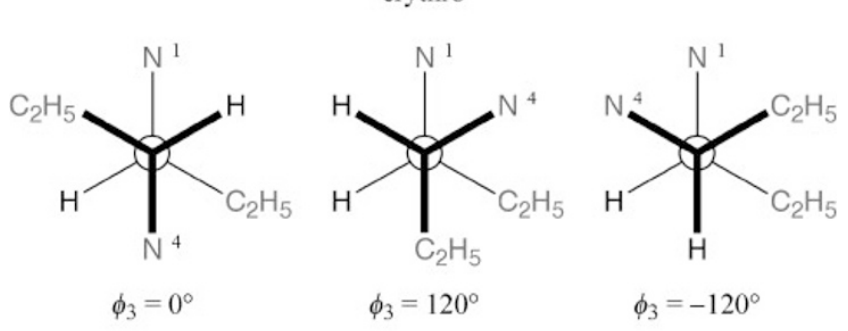

(b)

threo

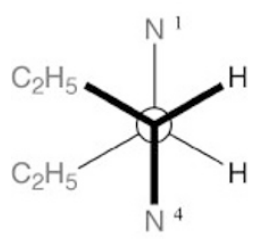

$\phi_{3}=0^{\circ}$

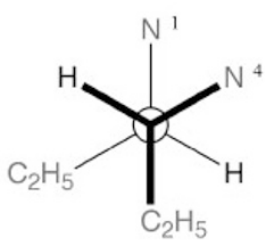

$\phi_{3}=120^{\circ}$

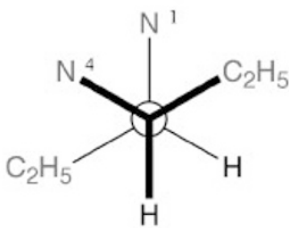

$\phi_{3}=-120^{\circ}$
Figure 7. Molecular structures of rotational isomers of erythro- (a) and threo-trans-pPrAz (b).

or

\begin{tabular}{crrcc}
\hline \multirow{2}{*}{ threo } & & \multicolumn{3}{c}{$\phi_{3}$} \\
\cline { 3 - 5 } & & $0^{\circ}$ & $120^{\circ}$ & $-120^{\circ}$ \\
\hline$\phi_{2}$ & $60^{\circ}$ & $\gamma$ & $\sigma \gamma$ & $\sigma$ \\
& $180^{\circ}$ & $\gamma$ & $\sigma \beta \gamma$ & $\sigma \beta$ \\
& $-60^{\circ}$ & $\gamma$ & $\sigma \gamma$ & $\sigma$ \\
\hline
\end{tabular}

Here, $\sigma$ is the statistical weight due to the steric hindrance between $\mathrm{N}^{1}$ and $\mathrm{N}^{4}$, which corresponds to the steric hindrance between $\mathrm{C}^{1}$ and $\mathrm{C}^{4}$ for diene polymers. (We do not consider the S,S-form of the threo unit because it is identical with the R,R-form in the calculation of $C_{N}$.) On the other hand, the above lower matrix for trans-diene polymers can be used as it is for trans-pPrAz. Thus, statistical properties of the rotational isomers for trans-pPrAz are described by the four parameters, $\alpha, \beta, \gamma$, and $\sigma$.

The population of the rotational isomers in each monomer unit of diene and azine polymers is independent of the state of the neighboring monomer units along the chain. In such a case, the characteristic ratio $C_{N}$ can be calculated by eq A.1 in Appendix. Mark ${ }^{17}$ demonstrated that the experimental characteristic ratio of trans-pBD is favorably compared with the RIS model with the statistical weights $\sigma=1, \alpha=0.96$, and $\beta=0.24$.

To calculate the $C_{N}$ for trans-pPrAz, eq A.1 must be extended for copolymers composed of the erythro and threo units. If the sequence of the erythro and threo units is random, the average with respect the erythro and threo isomers can be done independently along the copolymer chain, and eq A.1 is simply modified to eq A.7.

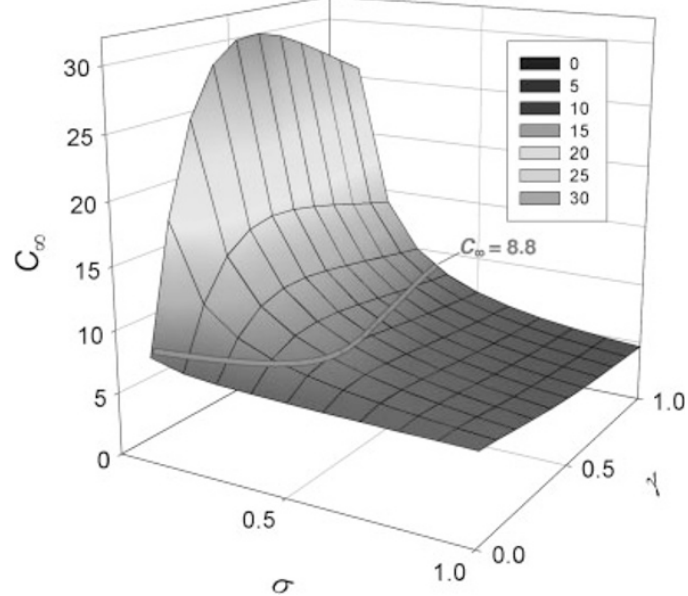

Figure 8. The characteristic ratio $C_{\infty}$ calculated by eq A.7 for trans-pPrAz as a function of $\sigma$ and $\gamma$ at fixed $\alpha=0.96$ and $\beta=$ 0.24 (see text).

It can be demonstrated that $C_{N}$ calculated by eq A.7 using reasonable values of the statistical weights quickly approaches to the asymptotic value $C_{\infty}$ with increasing $N$ ( $c f$. Figure S2 in the Supporting Information). Thus, we compare theoretical $C_{\infty}$ with experimental $C_{N}$ for trans-pPrAz with $N>50$ in what follows. Moreover, $C_{\infty}$ is dependent on $\alpha$ and $\beta$ relatively weakly as shown in Figure S3 in the Supporting Information, so that we may approximate the statistical weights $\alpha$ and $\beta$ for trans-pPrAz to those for transpBD.

Figure 8 shows $C_{\infty}$ for trans-pPrAz with the erythro fraction of 0.65 as a function of $\sigma$ and $\gamma$ at fixed $\alpha$ $(=0.96)$ and $\beta(=0.24)$. This figure exhibits that $\sigma$ must be less than $c a$. 0.5 to attain to the experimental values of $C_{N}(=8.8 \pm 0.4)$ in Table III. This is a contrast to the $\sigma$ value $(=1)$ of trans-pBD, ${ }^{17}$ and we can say that the more extended conformation of $\mathrm{pPrAz}$ arises from the difficulty of gauche conformation around bond 3 .

The characteristic ratio for photoisomerized $\mathrm{pPrAz}$ samples can be also calculated using eq A.7, where the samples are regarded as random copolymers of the trans and cis isomers. Quantities relating to the cis isomer in eq A.7 are estimated more simply using virtual bonds defined in Figure 9, as for cis-diene polymers. ${ }^{16}$ Since extensions of bonds 4 and 2 meet at a cross point $\mathrm{P}$ in the figure for the cis polymer, we can define two virtual bonds between $\mathrm{C}^{3}$ and $\mathrm{P}$ (bond a) and between $\mathrm{C}^{2}$ and $\mathrm{P}$ (bond $\mathrm{b}$ ), in place of bonds 4 and 2 in Figure 9. Bond 1 is not necessary to consider, and bond 3 is renamed as bond c. The bond length $b_{\mathrm{a}}\left(=b_{\mathrm{b}}\right)$ and the bond angle $\theta_{\mathrm{a}}$ were estimated to be $0.310 \mathrm{~nm}$ and $46.8^{\circ}$ by the Chem3D software.

In cis-pPrAz, ethyl groups attaching to $\mathrm{C}^{2}$ and $\mathrm{C}^{3}$ on both sides of the $\mathrm{N}=\mathrm{N}$ bond are close each other, 


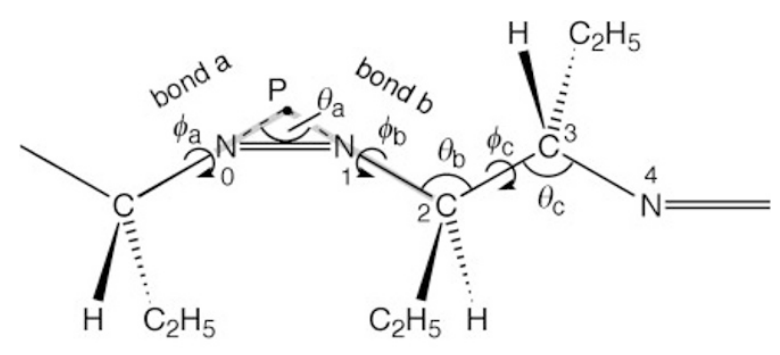

anti

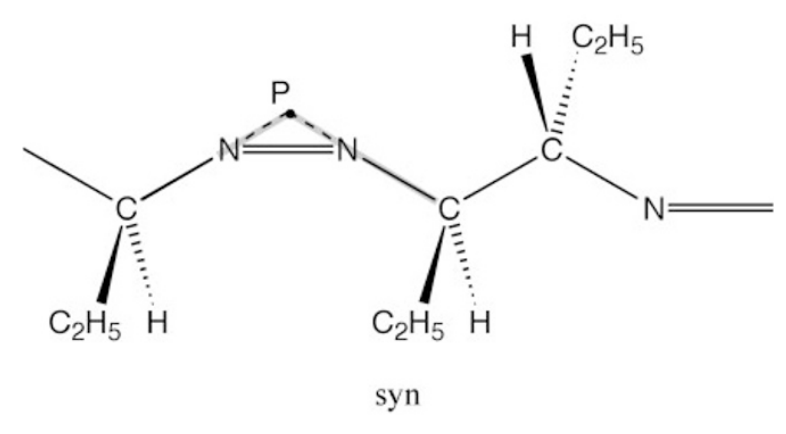

Figure 9. Molecular structures of anti- and syn-cis-pPrAz.

and restrict severely the rotations around bonds a and $b$; this effect was not necessary to be considered for cis-diene polymers. There are two stereo isomers with respect to the configuration of the two ethyl groups as also shown in Figure 9.22 For the anti unit, only two rotational states $\left(\phi_{\mathrm{a}}, \phi_{\mathrm{b}}\right)=\left(60^{\circ}, 60^{\circ}\right)$ and $\left(-60^{\circ},-60^{\circ}\right)$ are feasible to avoid severe steric hindrance between the ethyl groups. On the other hand, the feasible rotational state for the syn unit may be only $\left(60^{\circ},-60^{\circ}\right)$ or $\left(-60^{\circ}, 60^{\circ}\right)$. We can expect that bond $\mathrm{c}$ is independent of the rotational state of bond $\mathrm{b}$, and the statistical weights for bond $\mathrm{c}$ are the same as those for bond 3 in trans-pPrAz. For pPrAz with $f_{c i s}=0.33$, eq A.7 provides $C_{\infty} \approx 10.2$ and 4.7, if the cis unit is anti and syn ones, respectively. The experimental $C_{N}$ for photoisomerized pPrAz samples is close to the former. This indicates that the irradiation of $365 \mathrm{~nm}$ light preferentially produces the anticis unit, presumably because of the steric hindrance between ethyl groups on both sides of the $\mathrm{N}=\mathrm{N}$ bond.

Kamachi et al. $^{8}$ studied the variation in the chain size of poly(butyraldehyde azine) (pBuAz) with the trans-to-cis photoisomerization by size exclusion chromatography (SEC). Their result that the elution volume of photoirradiated $\mathrm{pBuAz}$ is larger than that of trans-pBuAz was opposite to our viscosity result for pPrAz. This implies that $C_{N}$ for azine polymers can sensitively depend on the chemical structure of the side chain. Unfortunately, Kamachi et al. did not measure absolute molecular weights of their $\mathrm{pBuAz}$ sample before and after photoisomerization, which is required to make a quantitative argument of $C_{N}$ for pBuAz.
Irie et al. ${ }^{3,4}$ reported the drastic effect of photoisomerization in polymers of which main chain is comprised of azobenzene units. The RIS model analysis demonstrates that the characteristic ratio $C_{N}$ of cisazine polymers strongly depends on the chemical structure of the side chains on both sides of azo group. Since the chemical structure on both sides of azo group is very much different between azine and azobenzene polymers, it is little wonder that the photoisomerization gives rise to the opposite change in the chain size of azobenzene polymers. The rigid phenylene unit attaching to azo group may enhance the change in the chain size by photoisomerization of azobenzene polymers. This effect is not expected for azine polymers.

Acknowledgment. This work was partly supported by a Grant-in-Aid for Scientific Research No. 17350058 from the Japan Society for the Promotion of Science.

\section{APPENDIX: CALCULATION OF THE CHARACTERISTIC RATIO}

When the monomer unit consists of four different kinds of main-chain bonds and the population of the rotational isomers is independent of the state of neighboring monomer units, the characteristic ratio $C_{N}$ can be calculated by

$$
C_{N}=1+\left(s+N^{-1} \boldsymbol{S}_{1} \boldsymbol{U}_{N} \boldsymbol{S}_{2}\right) / 2 \overline{b^{2}}
$$

where

$$
\begin{aligned}
s \equiv & \boldsymbol{b}_{1}{ }^{\mathrm{T}}\left\langle\boldsymbol{T}_{1}\right\rangle \boldsymbol{b}_{2}+\left(\boldsymbol{b}_{1}{ }^{\mathrm{T}}\left\langle\boldsymbol{T}_{1} \boldsymbol{T}_{2}\right\rangle+\boldsymbol{b}_{2}{ }^{\mathrm{T}}\left\langle\boldsymbol{T}_{2}\right\rangle\right) \boldsymbol{b}_{3} \\
& +\left(\boldsymbol{b}_{1}{ }^{\mathrm{T}}\left\langle\boldsymbol{T}_{1} \boldsymbol{T}_{2} \boldsymbol{T}_{3}\right\rangle+\boldsymbol{b}_{2}{ }^{\mathrm{T}}\left\langle\boldsymbol{T}_{2} \boldsymbol{T}_{3}\right\rangle+\boldsymbol{l}_{3}{ }^{\mathrm{T}}\left\langle\boldsymbol{T}_{3}\right\rangle\right) \boldsymbol{b}_{4}
\end{aligned}
$$

$$
\begin{aligned}
\boldsymbol{S}_{1} \equiv & \boldsymbol{b}_{1}^{\mathrm{T}}\left\langle\boldsymbol{T}_{1} \boldsymbol{T}_{2} \boldsymbol{T}_{3} \boldsymbol{T}_{4}\right\rangle+\boldsymbol{b}_{2}^{\mathrm{T}}\left\langle\boldsymbol{T}_{2} \boldsymbol{T}_{3} \boldsymbol{T}_{4}\right\rangle \\
& +\boldsymbol{b}_{3}^{\mathrm{T}}\left\langle\boldsymbol{T}_{3} \boldsymbol{T}_{4}\right\rangle+\boldsymbol{b}_{4}{ }^{\mathrm{T}}\left\langle\boldsymbol{T}_{4}\right\rangle \\
\boldsymbol{S}_{2} \equiv & \boldsymbol{b}_{1}+\left\langle\boldsymbol{T}_{1}\right\rangle \boldsymbol{b}_{2}+\left\langle\boldsymbol{T}_{1} \boldsymbol{T}_{2}\right\rangle \boldsymbol{b}_{3}+\left\langle\boldsymbol{T}_{1} \boldsymbol{T}_{2} \boldsymbol{T}_{3}\right\rangle \boldsymbol{b}_{4} \\
\boldsymbol{U}_{N} \equiv & {\left[(N-1) \boldsymbol{E}+\left(\boldsymbol{E}-\left\langle\boldsymbol{T}_{1} \boldsymbol{T}_{2} \boldsymbol{T}_{3} \boldsymbol{T}_{4}\right\rangle^{N-1}\right)\right.} \\
& \left.\times\left(\boldsymbol{E}-\left\langle\boldsymbol{T}_{1} \boldsymbol{T}_{2} \boldsymbol{T}_{3} \boldsymbol{T}_{4}\right\rangle^{-1}\right)^{-1}\right] \\
& \times\left(\boldsymbol{E}-\left\langle\boldsymbol{T}_{1} \boldsymbol{T}_{2} \boldsymbol{T}_{3} \boldsymbol{T}_{4}\right\rangle\right)^{-1}
\end{aligned}
$$

with the vector $\boldsymbol{b}_{i}$ of bond $i$ (a column vector), the unit matrix $\boldsymbol{E}$, and the transformation matrix $\boldsymbol{T}_{i}$ defined by

$$
\boldsymbol{T}_{i} \equiv\left(\begin{array}{ccc}
\cos \phi_{i} \cos \theta_{i} & \sin \phi_{i} & \cos \phi_{i} \sin \theta_{i} \\
\sin \phi_{i} \cos \theta_{i} & -\cos \phi_{i} & \sin \phi_{i} \sin \theta_{i} \\
\sin \theta_{i} & 0 & -\cos \theta_{i}
\end{array}\right)
$$

The superscript $\mathrm{T}$ indicates the transposition and $\langle\cdots\rangle$ means the statistical average taken with statistical weight matrices (see the text). 
Similarly, $C_{N}$ for the independent chain composed of 3 different kinds of main-chain bonds can be made by substituting $\boldsymbol{b}_{4}=0$ in eqs A.2-A.4 and taking out $\boldsymbol{T}_{4}$ from eqs A.3 and A.5.

For random copolymers, the average with respect the kind of the monomer unit can be done independently along the copolymer chain, and eq A.1 is simply modified to

$$
C_{N}=1+\left(s_{\mathrm{av}}+N^{-1} \boldsymbol{S}_{1, \mathrm{av}} \boldsymbol{U}_{N, \mathrm{av}} \boldsymbol{S}_{2, \mathrm{av}}\right) / 2 \overline{b^{2}}
$$

Here, the averaged quantity $X_{\mathrm{av}}\left(X=\mathrm{s}, \boldsymbol{S}_{1}, \boldsymbol{U}_{N}\right.$, or $\boldsymbol{S}_{2}$ ) is calculated for copolymers by $\sum_{j} f_{j} X_{j}$ with the fractions $f_{j}\left(\sum_{j} f_{j}=1\right)$ and the quantities $X_{j}$ for $j$ units (i.e., the erythro-trans, threo-trans, erythroanti-cis, erythro-syn-cis, threo-anti-cis, and threo-syncis units). At infinite $N, \boldsymbol{U}_{N}$ given by eq A.5 can be reduced to $N\left(\boldsymbol{E}-\left\langle\boldsymbol{T}_{1} \boldsymbol{T}_{2} \boldsymbol{T}_{3} \boldsymbol{T}_{4}\right\rangle\right)^{-1}$, and the calculation of eq A. 7 becomes much simpler.

Electronic Supporting Information Available: Figures S1, S2 and S3. These materials are available via. the Internet at http://www.spsj.or.jp/c5/pj/pj.htm

\section{REFERENCES}

1. For example: K. Kinbara and T. Aida, Chem. Rev., 105, 1377 (2005), and references therein.

2. For example: a) S. Yagai, T. Karatsu, and A. Kitamura, Chem. Eur. J., 11, 4054 (2005).

b) J.-P. Collin and J.-P. Sauvage, Chem. Lett., 34, 742 (2005).

3. M. Irie, Y. Hirano, S. Hashimoto, and K. Hayashi, Macromolecules, 14, 262 (1981).

4. M. Irie and W. Schnabel, Macromolecules, 14, 1246 (1981).

5. A. Harada, H. Fujii, and M. Kamachi, Macromolecules, 24, 5504 (1991).

6. A. Harada, A. Kajiwara, H. Fuji-i, and M. Kamachi, Polym. J., 24, 931 (1992).

7. M. Kamachi, A. Kajiwara, A. Hashidzume, K. Matsuda, and A. Harada, Des. Monomers Polym., 7, 701 (2004).
8. H. Fuji-i, A. Kajiwara, A. Harada, Y. Morishima, and M. Kamachi, Macromolecules, 25, 4224 (1992).

9. T. Curtius and E. Zinkeisen, J. Prakt. Chem., 58, 310 (1898).

10. H. Fujita, "Foundations of Ultracentrifugal Analysis," Wiley-Interscience, New York, 1975, Vol. 42.

11. M. Kurata, "Thermodynamics of Polymer Solutions," Harwood Academic Publishers: Chur, Switzerland, 1982.

12. As Kamachi et al. ${ }^{5-7}$ reported, resonance bands due to the methyl, methylene, and methine protons in pPrAz indicated the coexistence of isomeric units, i.e., erythro and threo units. From the ratio of area intensities, fractions were estimated to be $c a$. 0.65 and 0.35 . In the propagation reaction, the erythro unit may be formed preferentially because of less steric hindrance between the ethyl group on the active chain end and the ethyl group in the monomer. The rotational isomeric state model analysis supported the preferential formation of the erythro units. Thus, it can be concluded that the fractions of erythro and threo units are $c a .0 .65$ and 0.35 , respectively.

13. By subtracting Figure 2a from Figure 2b, the fractions of erythro and threo units after photoirradiation were confirmed to be practically the same as those before photoirradiation.

14. H. Yamakawa, "Helical Wormlike Chains in Polymer Solutions," Springer, Berlin, 1997.

15. R. H. Colby, L. J. Fetters, and W. W. Graessley, Macromolecules, 20, 2226 (1987).

16. J. E. Mark, J. Am. Chem. Soc., 88, 4654 (1966).

17. J. E. Mark, J. Am. Chem. Soc., 89, 6829 (1967).

18. H. Fujita, "Polymer Solutions," Elsevier, Amsterdam, the Netherlands, 1990, Vol. 9.

19. J. E. Kilpatrick and K. S. Pitzer, J. Res. Natl. Bur. Stand., 37, 163 (1946).

20. D. R. Lide, Jr. and D. E. Mann, J. Chem. Phys., 27, 868 (1957).

21. D. R. Lide, Jr. and H. Eyring, Annu. Rev. Phys. Chem., 15, 225 (1964).

22. Upon trans-to-cis photoisomerization, the syn- and antitrans units are converted into the anti- and syn-cis units, respectively, while the erythro and threo configurations are maintained. Thus, the fractions of erythro and threo units were fixed at 0.65 and 0.35 , respectively, upon calculation of $C_{\infty}$ for pPrAz after photoisomerization. 